\title{
Hepatocellular Carcinoma in Korea between 2012 and 2014: an Analysis of Data from the Korean Nationwide Cancer Registry
}

\author{
Young Eun Chon ${ }^{1 * \#}$, Han Ah Lee ${ }^{2^{*}}$, Jun Sik Yoon ${ }^{3^{*}}$, Jun Yong Park ${ }^{4}$, Bo Hyun Kim ${ }^{5}$, In Joon Lee ${ }^{6}$, Suk Kyun Hong , \\ Dong Hyeon Lee ${ }^{8}$, Hyun-Joo Kong', Eunyang Kim? \\ 'Department of Internal Medicine, CHA Bundang Medical Center, CHA University, Seongnam; 'Department of Internal Medicine, Korea \\ University College of Medicine, Seoul; ${ }^{3}$ Department of Internal Medicine, Busan Paik Hospital, Inje University College of Medicine, Busan; \\ ${ }^{4}$ Department of Internal Medicine, Yonsei University College of Medicine, Seoul; ${ }^{5}$ Center for Liver Cancer, Research Institute and Hospital, \\ National Cancer Center, Goyang; ${ }^{6}$ Department of Radiology, National Cancer Center, Goyang; ${ }^{7}$ Department of Surgery, Seoul National \\ University College of Medicine, Seoul; ${ }^{8}$ Department of Internal Medicine, Seoul Metropolitan Government Seoul National University \\ Boramae Medical Center, Seoul; ${ }^{9}$ Cancer Registration and Statistic Branch, National Cancer Control Institute, National Cancer Center, \\ Goyang; ${ }^{10}$ Department of Internal Medicine and Liver Research Institute, Seoul National University College of Medicine, Seoul, Korea
}

Received Jul. 29, 2020 Revised Aug. 14, 2020 Accepted Aug. 18, 2020
Background/Aims: Considering the high prevalence and mortality of hepatocellular carcinoma (HCC) in Korea, accurate statistics for HCC are important. We evaluated the characteristics of Korean patients with newly diagnosed HCC.

Methods: We retrospectively evaluated data from the Korean Primary Liver Cancer Registry (KPLCR). The baseline characteristics, treatment modalities, and overall survival (OS) of 4,572 patients with HCC registered in the KPLCR between 2012 and 2014 were investigated.

Results: At the time of HCC diagnosis, the median age was 60.0 years, with male predominance (79.6\%). Hepatitis B virus infection was the most common etiology (59.1\%). The rates of Barcelona Clinic Liver Cancer (BCLC) stages 0, A, B, C, and D at diagnosis were 3.9\%, 36.9\%, 12.5\%, 39.4\%, and $7.3 \%$, respectively. The proportion of very early or early stage HCC at diagnosis (BCLC stage 0 or A) in the 2012-2014 cohort was significantly lower than that in the 2008-2011 cohort (40.8\% vs. $48.3 \%, P<0.001)$. Transarterial therapy $(37.5 \%)$ was the most commonly performed initial treatment, followed by surgical resection (19.8\%), best supportive care (19.1\%), and local ablation (10.6\%). The median OS was 2.9 years, and the 1-, 3-, and 5-year OS rates were $67.7 \%, 49.3 \%$ and $41.9 \%$, respectively. The OS rate of the 2012-2014 cohort was significantly higher than that of the 2008-2011 cohort (log-rank, $P<0.001$ ).

Conclusions: The OS of HCC patients registered in the KPLCR between 2012 and 2014 significantly improved. Nevertheless, as about half of the HCC patients were diagnosed at an advanced stage, vigorous and optimized HCC screening strategies should be implemented. (J Liver Cancer 2020;20:135-147)

Keywords: Epidemiology; Hepatocellular carcinoma; Hepatitis B; Korea; Survival

\section{Corresponding author : Jeong-Hoon Lee}

Department of Internal Medicine, Seoul National University Hospital, 101 Daehak-ro, Jongno-gu, Seoul 03080, Korea

Tel. +82-2-2072-2228, Fax. +82-2-743-6701

E-mail; JHLeeMD@snu.ac.kr

https://orcid.org/0000-0002-0315-2080

\section{Co-corresponding author: Young Eun Chon}

Department of Internal Medicine, CHA Bundang Medical Center, CHA University, 59 Yatap-ro, Bundang-gu, Seongnam 13496, Korea

Tel. +82-31-780-2947, Fax. 82-31-780-2949

E-mail; nachivysoo@chamc.co.kr

https://orcid.org/0000-0002-7716-4850

* Young Eun Chon, Han Ah Lee, and Jun Sik Yoon contributed equally to this work as co-first authors.

\# Jeong-Hoon Lee and Young Eun Chon contributed equally to this work as co-corresponding authors.

Copyright ( $\odot 2020$ by The Korean Liver Cancer Association. All rights reserved.

This is an Open Access article distributed under the terms of the Creative Commons Attribution Non-Commercial License (http://creativecommons.org/licenses/by-nc/4.0/) which permits unrestricted non-commercial use, distribution, and reproduction in any medium, provided the original work is properly cited. 


\section{INTRODUCTION}

Liver cancer is the second most common cause of cancerrelated deaths worldwide, with almost 800,000 deaths annually. ${ }^{1}$ Hepatocellular carcinoma (HCC) accounts for more than $80 \%$ of all liver cancers, representing a major global health problem. ${ }^{2}$ In South Korea, liver cancer is the sixth most common cancer and the second leading cause of cancer-related deaths. ${ }^{3}$ Hepatitis B virus (HBV) infection is the major etiology of HCC attributable to $62-75 \%$ of total cases. In an effort to reduce HBV infection, a nationwide vaccination project has been successfully implemented to enhance HBV prevention, and antiviral treatment has been widely spread to control viral replications. ${ }^{5}$ Owing to this effort, the prevalence of chronic hepatitis $\mathrm{B}$ and liver disease related mortality has been significantly reduced, and the age-standardized incidence rate of HCC has shown a gradually decreasing trend since $2000 .{ }^{3}$ Nonetheless, as the life expectancy and the likelihood of developing HCC increase in patients with $\mathrm{HBV}$, the socioeconomic burden of HCC is expected to increase. $^{6}$

HCC screening in high risk patients is known to improve the prognosis of HCC through the early detection of tumor and application of curative treatments. ${ }^{7}$ A national HCC surveillance program with ultrasonography and serum alpha-fetoproetin (AFP) test at 6-month intervals for patients at high risk for HCC has been established in Korea since 2003. ${ }^{8}$ The participation rate for this surveillance program has increased annually from 2003 to $2012 .{ }^{9}$ One of the most important issues worth studying might be whether patient survival has increased in correlation with the implementation of the national HCC surveillance program.

Regarding HCC treatment, various modalities and multidisciplinary approaches have been introduced. ${ }^{10,11}$ Sorafenib, a multi-kinase inhibitor was first introduced in Korea as a systemic treatment for unresectable HCC in 2008, and its prescriptions have increased over time with the gradual expansion of insurance coverage. ${ }^{12}$ Radiation therapy has also been recognized as a treatment for HCC since the early 2000s, and is performed solely or in combination with transarterial therapy or systemic treatment nowadays. ${ }^{13}$ Therefore, the prognosis and survival of HCC patients may have changed with changes in HCC characteristics and treatments over time.

The Korean Primary Liver Cancer Registry (KPLCR) is a nationwide population-based cancer registry, and the samples registered here are guaranteed to be representative of HCC in Korea. Based on these data, we have published a paper on HCC statistics in Korea previously. ${ }^{14}$ In the present study, we investigated the characteristics (clinical and tumor characteristics, treatment modalities, and survival) of Korean patients with newly diagnosed HCC between 2012 and 2014.

\section{METHODS}

\section{Patients}

Patients were selected from the KPLCR between January 2012 and December 2014. Details of the KPLCR have been described in our previous article. ${ }^{14}$ Briefly, the KPLCR is a random sample consisting of $15 \%$ of newly diagnosed HCC patients in Korea, which represents the characteristics of HCC of Korea. HCC was diagnosed based on histological evidence or via dynamic computed tomography (CT) and/or magnetic resonance imaging (MRI) findings (nodule $>1 \mathrm{~cm}$ with arterial hypervascularity and portal-/delayed-phase washout). ${ }^{10,11}$

Between 2012 and 2014, 4,593 patients were registered in the KPLCR. Of these, 21 patients were histologically diagnosed with other malignancies and initially excluded from the study. Among the remaining 4,572 patients with HCC, we excluded 1) patients who received their initial treatment more than 120 days from the date of diagnosis $(n=72)$ and 2$)$ patients lacking information on treatment modalities $(n=4)$. Finally, a total of 4,496 HCC patients was selected as the treatment group. The treatment group consisted of patients who received active treatment $(n=3,639)$ and those who received best supportive care $(\mathrm{n}=857)$. Supplementary Fig. 1 shows a flow chart of patient selection in the present study. Seventy-two patients who received an initial treatment more than 120 days after the first HCC diagnosis were initially excluded from the treatment group and subsequent survival 
analyses because they were likely to receive the treatment at a more advanced tumor stage than their initial tumor stage. ${ }^{15}$ The need for institutional review board approval and written informed consent were waived because the KPLCR data were collected as part of the Korean Central Cancer Registry (KCCR) in accordance with the Cancer Control Act.

\section{Data collection and definitions}

Patient information was obtained from the medical records of each hospital where the HCC diagnosis was made. Well-trained KCCR registry record personnel at each hospital investigated the medical records. Data were extracted using a standardized case record form and validated by statisticians at the KCCR and KPLCR. The data included baseline characteristics such as demographic, laboratory, and tumor variables, and treatment factors, including treatment modalities and overall survival (OS) of the patients. All tumor characteristics were assessed by diagnostic imaging such as dynamic CT or MRI scans. The modified Union for International Cancer Control (mUICC) ${ }^{16}$ and Barcelona Clinic Liver Cancer (BCLC) staging systems ${ }^{17}$ were adapted for HCC staging. The OS was measured from the date of HCC diagnosis until death from any cause. Death certificate information was obtained from national statistical data collected by the Korean Ministry of Government Administration and Home Affairs. Individual patients' vital statistics data were identified using 13-digit unique resident registration numbers issued to all Koreans. The data cutoff date was December 31, 2017. For comparative analyses with the previous period, data of the 2008-2011 cohort $(n=5,974)$ were extracted from the KPLCR. ${ }^{14}$ Data of the 2003-2005 cohort $(\mathrm{n}=4,520)$ were extracted from the nationwide HCC cohort of the Korean Liver Cancer Study Group which was founded in 1999 by a group of leading liver specialists in Korea. ${ }^{18}$

\section{Statistical analysis}

The data are presented as numbers (percentages) or medians (interquartile ranges [IQRs]), as appropriate. The significance of differences among continuous and categorical vari- ables was examined using the student $t$-test (or MannWhitney $U$ test) and chi-squared test (or Fisher's exact test), respectively. The OS of patients was evaluated using the Kaplan-Meier method, and the survival differences were compared by using the log-rank test. A Cox regression analysis was performed to assess the association between OS and the variables, and to calculate hazard ratios (HRs) and their 95\% confidence intervals (CIs). All statistical analyses were performed using SAS version 9.4 (SAS Institute Inc., Cary, NC, USA) and R version 3.5.2 (The R Project for Statistical Computing, Vienna, Austria). Two-sided $P$-values $<0.05$ were considered to indicate statistical significance.

\section{RESULTS}

\section{Baseline characteristics}

The baseline characteristics of the study population (20122014; $\mathrm{n}=4,572$ ) are summarized in Table 1 . The median age was 60 years (IQR, 53-70 years) with male predominance (79.6\%). HBV was the most common etiology (59.1\%), followed by alcohol (13.5\%), hepatitis C virus (10.8\%), and others $(16.8 \%)$. With regard to liver function, $72.9 \%, 22.4 \%$, and $4.7 \%$ of patients were classified into Child-TurcottePugh (CTP) classes A, B, and C, respectively, and the median Model for End stage Liver Disease (MELD) score was 8.0. The median AFP level was $30.7 \mathrm{ng} / \mathrm{mL}$ (IQR, 5.8-679.7 ng/ $\mathrm{mL})$. According to the mUICC staging system, stage II (36.6\%) was most common, followed by stage III (25.7\%), stage I (15.0\%), stage IV-A (11.8\%), and stage IV-B (10.1\%). According to the BCLC staging system, stage C (39.4\%) was most common, followed by stage A (36.9\%), stage B (12.5\%), stage D (8.6\%), and stage 0 (3.9\%). Solitary tumors were observed among $60.0 \%$ of the patients, and the median maximum tumor diameter was $3.4 \mathrm{~cm}$ (IQR, 2.0-6.8 cm). Macroscopic portal vein and hepatic vein invasions were detected in $23.3 \%$ and $5.7 \%$ of patients, respectively. Bile duct invasion was noticed in $2.2 \%$ of patients. Lymph node metastasis and distant metastasis were observed in 7.3\% and $10.1 \%$ of patients, respectively.

We compared the baseline characteristics of the study 
Table 1. Baseline characteristics of the study population (2012-2014; $n=4,572)$ and comparison with the data from 2008-2011 $(n=6,028)$

\begin{tabular}{|c|c|c|c|}
\hline Variable & $2012-2014$ & $2008-2011$ & $P$-value \\
\hline \multicolumn{4}{|l|}{ Demographic variable } \\
\hline Age (years) & $60.0(53.0-76.0)$ & $59.0(51.0-68.0)$ & $<0.001$ \\
\hline Sex (male) & 3,638 (79.6) & $4,790(78.7)$ & 0.310 \\
\hline Diabetes & $1,197(26.4)$ & $1,401(23.0)$ & 0.001 \\
\hline Hypertension & $1,648(36.4)$ & $1,808(29.7)$ & $<0.001$ \\
\hline Body mass index $\left(\mathrm{kg} / \mathrm{m}^{2}\right)$ & $23.7(21.5-25.9)$ & $23.7(21.7-25.8)$ & 0.800 \\
\hline Smoking & $2,069(45.7)$ & $2,657(43.7)$ & 0.240 \\
\hline Etiology & $(n=4,572$, missing $=0)$ & $(n=5,291$, missing $=792)$ & $<0.001$ \\
\hline $\mathrm{HBV}^{\dagger}$ & $2,700(59.1)$ & $3,810(72.0)$ & \\
\hline $\mathrm{HCV}$ & $490(10.7)$ & $636(12.0)$ & \\
\hline Alcohol & $616(13.5)$ & $711(13.4)$ & \\
\hline Others & $766(16.8)$ & $134(2.5)$ & \\
\hline Performance status* & $(n=3,114$, missing $=1,458)$ & $(\mathrm{n}=4,162$, missing $=1,921)$ & $<0.001$ \\
\hline 0 & $2,224(71.4)$ & $3,231(77.6)$ & \\
\hline 1 & $665(21.4)$ & $641(15.4)$ & \\
\hline 2 & $141(4.5)$ & $159(3.8)$ & \\
\hline 3 & $62(2.0)$ & $74(1.8)$ & \\
\hline 4 & $22(0.7)$ & $57(1.4)$ & \\
\hline Ascites & $(n=4,566$, missing $=6)$ & $(n=6,006$, missing $=77)$ & 0.610 \\
\hline Absent & $3,462(75.8)$ & $4,509(75.1)$ & \\
\hline Mild & 701 (15.4) & $964(16.1)$ & \\
\hline Moderate to severe & $403(8.8)$ & $533(8.9)$ & \\
\hline Encephalopathy & $(n=4,567$, missing $=5)$ & $(n=6,045$, missing $=38)$ & 0.270 \\
\hline None & $4,468(97.8)$ & $5,894(97.5)$ & \\
\hline Mild to moderate (grade 1 or 2) & $84(1.8)$ & $119(2.0)$ & \\
\hline Severe (grade 3 or 4 ) & $15(0.3)$ & $32(0.5)$ & \\
\hline \multicolumn{4}{|l|}{ Laboratory variable } \\
\hline Total bilirubin (mg/dL) & $0.9(0.6-1.5)$ & $1.0(0.70-1.60)$ & $<0.001$ \\
\hline Serum albumin $(g / d L)$ & $3.8(3.3-4.3)$ & $3.8(3.2-4.2)$ & $<0.001$ \\
\hline Alanine aminotransferase (IU/L) & $35.0(23.0-57.5)$ & $38.0(24.0-61.0)$ & $<0.001$ \\
\hline Platelet count $\left(10^{9} / \mathrm{L}\right)$ & $144.0(100.0-196.0)$ & $139.0(95.0-199.0)$ & 0.151 \\
\hline Prothrombin time (INR) & $1.10(1.03-1.21)$ & $1.11(1.04-1.22)$ & 0.020 \\
\hline Creatinine (mg/dL) & $0.88(0.73-1.02)$ & $0.90(0.74-1.00)$ & 0.305 \\
\hline Sodium (mmol/L) & $139.0(136.0-141.0)$ & $139.0(136.0-141.0)$ & 0.482 \\
\hline Glucose (mg/dL) & $110.0(95.0-142.0)$ & $109.0(94.0-139.0)$ & 0.200 \\
\hline Total cholesterol (mg/dL) & $156.0(131.0-183.0)$ & $155.0(130.0-182.0)$ & 0.252 \\
\hline Child-Turcotte-Pugh class & $(n=4,425$, missing $=147)$ & $(n=5,719$, missing $=364)$ & $<0.001$ \\
\hline A & $3,226(72.9)$ & $4,063(71.0)$ & \\
\hline B & $991(22.4)$ & $1,362(23.8)$ & \\
\hline C & $208(4.7)$ & $294(5.1)$ & \\
\hline MELD score & $8.0(7.0-1.0)$ & $8.6(7.3-11.1)$ & $<0.001$ \\
\hline MELD-Na score & $10.0(8.0-13.0)$ & $10.1(8.0-13.8)$ & 0.003 \\
\hline
\end{tabular}




\begin{tabular}{|c|c|c|c|}
\hline Variable & $2012-2014$ & $2008-2011$ & $P$-value \\
\hline \multicolumn{4}{|l|}{ Tumor variable } \\
\hline Alpha-fetoprotein (ng/mL) & $30.7(5.8-679.7)$ & $47.4(7.3-785.0)$ & $<0.001$ \\
\hline PIVKA-II (mAU/mL) & $125.0(27.0-2,228.0)$ & $144.5(30.0-1,373.0)$ & 0.008 \\
\hline Numbers of tumors & $(n=4,552$, missing $=20)$ & $(n=6,161$, missing $=22)$ & 0.624 \\
\hline 1 & $2,729(60.0)$ & $3,725(61.5)$ & \\
\hline 2 & $646(14.2)$ & $826(13.6)$ & \\
\hline 3 & $186(4.1)$ & $247(4.1)$ & \\
\hline 4 & $74(1.6)$ & $95(1.6)$ & \\
\hline$\geq 5$ & $917(20.1)$ & $1,168(19.3)$ & \\
\hline Maximum tumor diameter $(\mathrm{cm})$ & $3.4(2.0-6.8)$ & $3.0(2.0-5.4)$ & $<0.001$ \\
\hline Portal vein invasion & $1,061(23.3)$ & $1,477(24.3)$ & 0.242 \\
\hline Hepatic vein invasion & $261(5.7)$ & $339(5.6)$ & 0.498 \\
\hline Bile duct invasion & $100(2.2)$ & $159(2.6)$ & 0.191 \\
\hline Lymph node metastasis & $331(7.3)$ & $431(7.1)$ & 0.492 \\
\hline Distant metastasis & $460(10.1)$ & $602(9.9)$ & 0.490 \\
\hline Modified UICC Stage & $(n=4,534$, missing $=38)$ & $(n=6,045$, missing $=38)$ & 0.643 \\
\hline Stage I & $685(15.0)$ & $854(14.1)$ & \\
\hline Stage II & $1,674(36.6)$ & $2,267(37.5)$ & \\
\hline Stage III & $1,174(25.7)$ & $1,577(26.1)$ & \\
\hline Stage IV-A & $541(11.8)$ & $746(12.3)$ & \\
\hline Stage IV-B & $460(10.1)$ & 601 (9.9) & \\
\hline $\mathrm{BCLC}$ stage & $(n=3,727$, missing $=845)$ & $(n=5,697$, missing $=386)$ & $<0.001$ \\
\hline 0 & $145(3.9)$ & $489(8.6)$ & \\
\hline A & $1,376(36.9)$ & $2,260(39.7)$ & \\
\hline B & $465(12.5)$ & $658(11.5)$ & \\
\hline$C$ & 1,470 (39.4) & 1,923 (33.8) & \\
\hline $\mathrm{D}$ & $271(7.3)$ & $391(6.9)$ & \\
\hline
\end{tabular}

The values are presented as the median (interquartile range) or number (\%).

HBV, hepatitis B virus; HCV, hepatitis C virus; INR, international normalized ratio; MELD, Model for End stage Liver Disease; PIVKA-II, protein induced by vitamin K absence-II; UICC, Union for International Cancer Control; BCLC, Barcelona Clinic Liver Cancer.

"Performance status was classified as follows: 0 , fully active without symptoms; 1 , ambulatory with symptoms; 2 , bedridden $<50 \%$ of the time; 3 , bedridden $>50 \%$ of the time but capable of self-care; 4 , bedridden $100 \%$ of the time and incapable of self-care; ${ }^{\dagger}$ Patients co-infected with HBV and HCV ( $n=87)$ were also included.

populations of 2012-2014 vs. 2008-2011. Patients in the 2012-2014 study population were significantly older (60 years vs. 59 years), and had lower proportion of HBV etiology (59.1\% vs. $72.0 \%)$, CTP class B or C (27.1\% vs. $28.9 \%)$, MELD score (8.0 vs. 8.6), and AFP level (30.7 ng/mL vs. 47.4 $\mathrm{ng} / \mathrm{mL})$. However, patients in the 2012-2014 cohort had a higher proportion of poor performance status ( $\geq 1: 28.6 \%$ vs. $22.4 \%$ ), advanced stage (BCLC stage C or D: $46.7 \%$ vs. $40.7 \%)$, and had larger maximal diameter $(3.4 \mathrm{~cm}$ vs. $3.0 \mathrm{~cm})$ (all $P<0.05)$.

\section{Initial treatment modality}

Table 2 presents the distribution of initial treatment modalities in the treatment group $(n=4,496)$. The most common treatment modality was transarterial therapy $(37.5 \%)$, followed by surgical resection (19.8\%), best supportive care (19.1\%), and local ablation therapy (10.6\%). A majority of 
Table 2. Distribution of initial treatment modalities among patients in the treatment groups in 2012-2014 ( $n=4,496)$ and 2008-2011 ( $n=5,974)$

\begin{tabular}{|c|c|c|}
\hline Treatment modality & 2012-2014 & 2008-2011 \\
\hline Surgical resection & $890(19.8)$ & $998(16.7)$ \\
\hline Liver transplantation & $51(1.1)$ & $55(0.9)$ \\
\hline Local ablation therapy & $476(10.6)$ & $636(10.6)$ \\
\hline RFA & 440 & 594 \\
\hline PEI & 35 & 37 \\
\hline Other local ablation & 1 & 5 \\
\hline Transarterial therapy & $1,685(37.5)$ & $2,493(41.7)$ \\
\hline Conventional TACE & 1,527 & 2,407 \\
\hline TACE with drug-eluting beads & 99 & 64 \\
\hline Radioembolization & 21 & 7 \\
\hline HAIC & 38 & 82 \\
\hline Combination therapy* & $113(2.5)$ & $108(1.8)$ \\
\hline Systemic therapy & $228(5.1)$ & $224(3.7)$ \\
\hline Sorafenib & 205 & 175 \\
\hline Other systemic agents & 23 & 77 \\
\hline External beam radiation therapy & $66(1.5)$ & $61(1.0)$ \\
\hline Best supportive care & $857(19.1)$ & $1,295(21.7)$ \\
\hline Miscellaneous therapies ${ }^{\dagger}$ & $130(2.9)$ & $104(1.7)$ \\
\hline
\end{tabular}

The values are presented as number (\%) unless otherwise indicated.

RFA, radiofrequency ablation; PEI, percutaneous ethanol injection; TACE, transarterial chemoembolization; HAIC, hepatic arterial infusion chemotherapy.

${ }^{*}$ Combination therapy was defined as combined treatment with local ablation therapy and transarterial therapy; ${ }^{\dagger}$ Miscellaneous therapies were defined as unclassifiable treatment modalities (i.e., combination therapies other than transarterial therapy and local ablation therapy).

patients who underwent local ablation therapy received radiofrequency ablation (440 of 476, 92.4\%). Among patients who underwent transarterial therapies, conventional transarterial chemoembolization was the most frequently performed procedure $(1,527$ of $1,685,96.6 \%)$. Sorafenib was the most common systemic therapeutic agent (175 of $224,78.1 \%$ ). There were significant differences in the trends of initial treatment modality between the patients of the 2012-2014 and 2008-2011 cohorts $(P<0.001)$. Compared to the 20082011 cohort, the proportion of patients treated with transarterial therapy in the 2012-2014 cohort decreased (from $41.7 \%$ to $37.5 \%$ ), and the proportion of patients who underwent surgical resection (from $16.7 \%$ to $19.8 \%$ ) or liver transplantation (from $0.9 \%$ to $1.1 \%$ ) increased. The proportion of patients who underwent systemic therapy (from 3.7\% to $5.1 \%$ ) or external beam radiation therapy (from $1.0 \%$ to $1.5 \%$ ) also increased (all $P<0.05$ ).
The distribution of treatment modalities, according to the BCLC stage, is shown in Fig. 1. Among patients with BCLC stage 0 , local ablation therapy was most widely performed (47.6\%). For HCC of BCLC stages A, B, and C, transarterial therapy was the most common mode of treatment at each stage (36.8\% in BCLC A, $53.3 \%$ in BCLC B, and $35.6 \%$ in BCLC C). Most patients with BCLC stage D, received best supportive care (59.9\%). When we investigated the adherence rate of the real-world HCC treatment practice in comparison with the BCLC treatment guideline, the overall adherence rate of the total study population was $38.2 \%$. The rates of adherence to the BCLC treatment guideline according to each BCLC stage are depicted in Supplementary Table 1. For patients with BCLC stage $\mathrm{C}$, although systemic treatment (i.e., sorafenib) is the recommended method of treatment, the adherence rate was merely $11.7 \%$. 


\section{Total}

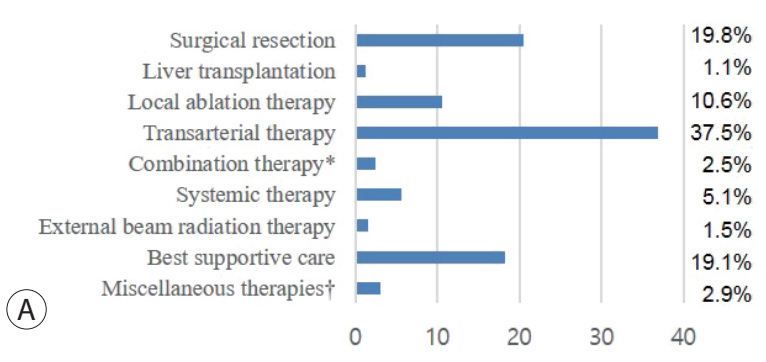

BCLC Stage A

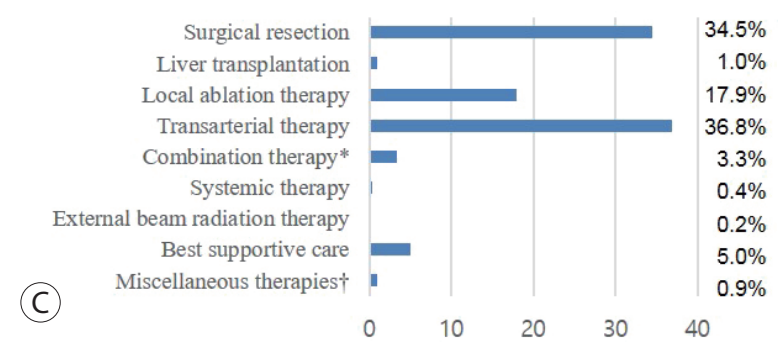

BCLC Stage C

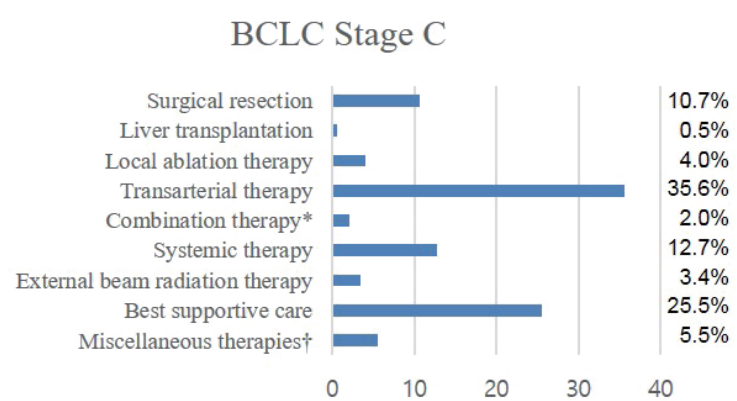

\section{BCLC Stage 0}

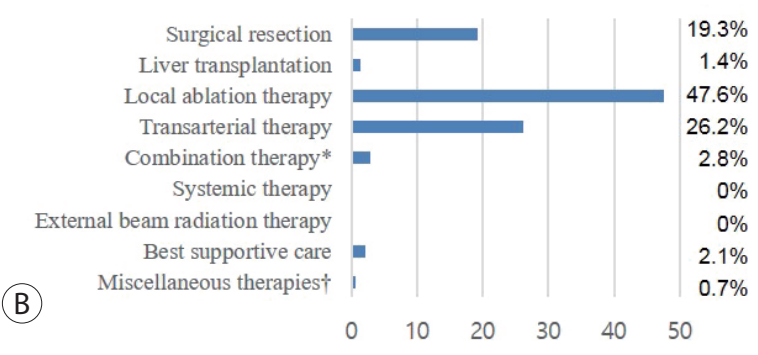

\section{BCLC Stage B}

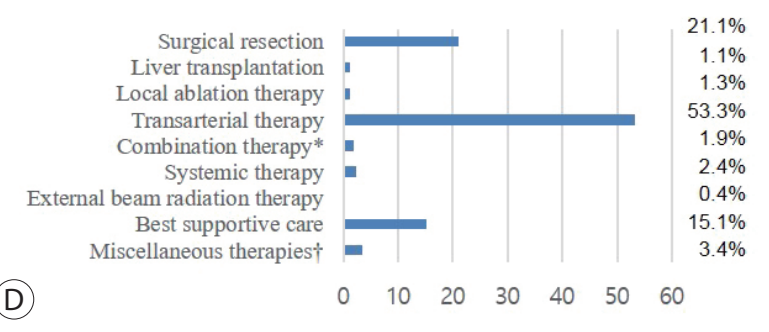

\section{BCLC Stage D}

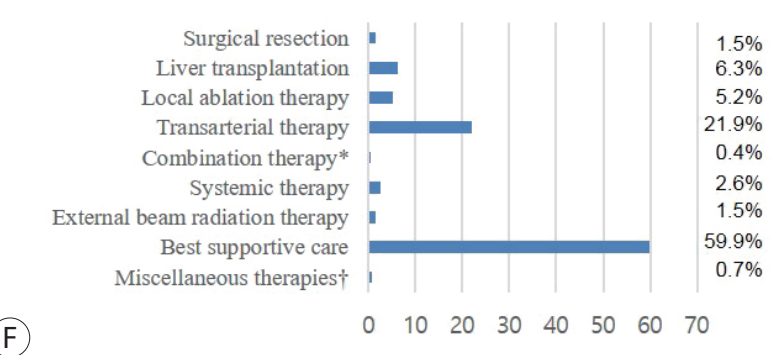

Figure 1. Initial treatment modalities of the study population (A) and subgroups according to the Barcelona Clinic Liver Cancer (BCLC) stage (B), stage $A(C)$, stage $B(D)$, stage $C(E)$, and stage $D(F)$. ${ }^{*}$ Combination therapy was defined as combined treatment with local ablation transarterial therapy; ${ }^{\dagger}$ Miscellaneous therapies were defined as unclassifiable treatment modalities (i.e., combination therapies other than transarterial therapy and local ablation therapy).

\section{Survival analyses}

Table 3 presents the median OS and annual OS rates of the entire study population, and the OS rates according to the baseline characteristics. The median OS was 2.90 years (IQR, 2.64-3.11 years), and the 1-, 3-, and 5-year OS rates were $67.7 \%, 49.3 \%$, and $41.9 \%$, respectively. The OS rate of the 2012-2014 cohort was significantly higher than that of the 2008-2011 cohort as well as the 2003-2005 cohort (both logrank tests, $P<0.001$ ) (Fig. 2). The unadjusted HR of death was 0.64 (95\% CI, $0.61-0.68 ; P<0.001)$ in the $2012-2014$ cohort compared with the 2003-2005 cohort. The unadjusted
HR of death was $0.75(95 \% \mathrm{CI}, 0.72-0.79, P<0.001)$ in the 2008-2011 cohort compared with the 2003-2005 cohort. All of the survival curves according to CTP class (Fig. 3A), mUICC stage (Fig. 3B), BLCL stage (Fig. 3C), and AFP level (Fig. 3D) showed significant differences in survival (all logrank tests, $P<0.001)$. We also performed post hoc analyses for multiple comparisons between each survival curve and found significant survival differences between all CTP classes, between all HCC stages, and between all AFP levels (all pairwise comparisons using log-rank tests, $P<0.001$ ). 
Table 3. Overall survival rates (\%) of the study population according to baseline characteristics and initial treatment modalities

\begin{tabular}{|c|c|c|c|c|c|c|c|}
\hline \multirow{2}{*}{ Variable } & \multirow{2}{*}{ Value } & \multirow{2}{*}{$\begin{array}{c}\text { Median OS } \\
\text { (years, } 95 \% \mathrm{Cl} \text { ) }\end{array}$} & \multicolumn{5}{|c|}{ Year } \\
\hline & & & 1 & 2 & 3 & 4 & 5 \\
\hline All patients & $4,572(100)$ & $2.90(2.64-3.11)$ & 67.7 & 56.6 & 49.3 & 44.6 & 41.9 \\
\hline \multicolumn{8}{|c|}{ Age (years; $n=4,572$, missing values $=0$ ) } \\
\hline$<40$ & $136(3.0)$ & $2.70(1.26-5.48)$ & 61.8 & 53.7 & 47.1 & 44.1 & 42.3 \\
\hline $40-49$ & $557(12.2)$ & $2.99(2.27-4.25)$ & 65.2 & 56 & 49.9 & 46.1 & 43.9 \\
\hline $50-59$ & $1,486(32.5)$ & $4.18(3.50-5.94)$ & 69.9 & 61 & 54.8 & 50.8 & 48 \\
\hline $60-69$ & $1,209(26.4)$ & $4.19(3.54-5.37)$ & 72.9 & 62.5 & 54.7 & 50.7 & 47.7 \\
\hline$\geq 70$ & $1,184(25.9)$ & $1.65(1.50-1.87)$ & 61.7 & 45.8 & 37 & 29.8 & 27.4 \\
\hline \multicolumn{8}{|c|}{ Sex $(n=4,572$, missing values $=0)$} \\
\hline Male & $3,638(79.6)$ & $2.67(2.43-2.92)$ & 66.6 & 55.2 & 47.7 & 43.3 & 40.5 \\
\hline Female & $934(20.4)$ & $3.92(3.22-5.47)$ & 72.1 & 62.2 & 55.6 & 49.6 & 47.3 \\
\hline \multicolumn{8}{|c|}{ Etiology $(n=4,572$, missing values $=0$ ) } \\
\hline $\mathrm{HBV}^{*}$ & $2,700(59.1)$ & $3.64(3.12-4.34)$ & 68.2 & 58.9 & 52.4 & 49.0 & 46.5 \\
\hline $\mathrm{HCV}$ & $490(10.7)$ & $2.56(2.15-3.06)$ & 72.4 & 55.7 & 46.7 & 38.0 & 34.5 \\
\hline Alcohol & $616(13.5)$ & $2.46(2.04-2.88)$ & 68.0 & 54.5 & 45.0 & 39.4 & 36.4 \\
\hline Others & $766(16.8)$ & $2.04(1.68-2.44)$ & 62.1 & 50.3 & 43.0 & 37.1 & 35.0 \\
\hline \multicolumn{8}{|c|}{$\begin{array}{l}\text { Child-Turcotte-Pugh classification ( } n=4,425 \text {, } \\
\text { missing values }=147 \text { ) }\end{array}$} \\
\hline A & $3,226(72.9)$ & NR (5.24-NR) & 79.7 & 68.4 & 60.6 & 55.5 & 52.1 \\
\hline B & $991(22.4)$ & $0.57(0.49-0.68)$ & 39.1 & 27.9 & 21.6 & 17.6 & 16.6 \\
\hline C & $208(4.7)$ & $0.25(0.20-0.33)$ & 26.4 & 19.2 & 13.9 & 12.5 & 11.1 \\
\hline \multicolumn{8}{|c|}{$\begin{array}{l}\text { Alpha-fetoprotein ( } \mathrm{ng} / \mathrm{mL} ; \mathrm{n}=4,197 \text {, missing } \\
\text { values=375) }\end{array}$} \\
\hline$<11.45$ & $1,599(38.1)$ & NR (NR-NR) & 85.2 & 74.5 & 66.4 & 61.3 & 58.2 \\
\hline $11.45-19.99$ & $314(7.5)$ & $4.36(3.61-5.15)$ & 77.1 & 66.6 & 59.6 & 52.2 & 46.4 \\
\hline 20.00-99.99 & $653(15.6)$ & $3.48(2.99-4.31)$ & 75.0 & 62.6 & 53.4 & 47.6 & 44.6 \\
\hline $100.00-199.99$ & $217(5.2)$ & $3.24(2.28-4.77)$ & 71.4 & 60.4 & 52.5 & 45.2 & 42.2 \\
\hline 200.00-399.99 & $226(5.4)$ & $2.17(1.34-2.68)$ & 62.4 & 51.8 & 42.0 & 36.7 & 33.7 \\
\hline$\geq 400.00$ & 1,188 (28.3) & $0.61(0.55-0.68)$ & 39.0 & 28.3 & 23.7 & 21.5 & 20.4 \\
\hline \multicolumn{8}{|c|}{$\begin{array}{l}\text { Modified UICC stage ( } n=4,534 \text {, missing } \\
\text { values }=38)\end{array}$} \\
\hline Stage I & $685(15.0)$ & NR (NR-NR) & 93.9 & 87.9 & 81.8 & 76.6 & 74.0 \\
\hline Stage II & 1,674 (36.6) & NR (NR-NR) & 87.0 & 76.9 & 68.7 & 62.7 & 59.1 \\
\hline Stage III & $1,174(25.7)$ & $1.85(1.63-2.07)$ & 64.4 & 48.0 & 37.9 & 32.7 & 29.5 \\
\hline Stage IV-A & $541(11.8)$ & $0.44(0.39-0.50)$ & 29.8 & 16.6 & 13.1 & 10.7 & 9.3 \\
\hline Stage IV-B & $460(10.1)$ & $0.27(0.25-0.31)$ & 13.5 & 6.1 & 3.7 & 2.0 & 2.0 \\
\hline \multicolumn{8}{|c|}{ BCLC stage $(n=3,727$, missing values $=845)$} \\
\hline 0 & 145 (3.9) & NR (NR-NR) & 97.2 & 93.8 & 91.0 & 86.2 & 83.4 \\
\hline A & $1,376(36.9)$ & NR (NR-NR) & 92.7 & 83.6 & 76.3 & 71.0 & 66.8 \\
\hline B & $465(12.5)$ & $2.81(2.39-3.29)$ & 75.7 & 59.8 & 48.4 & 40.9 & 36.7 \\
\hline C & $1,470(39.4)$ & $0.75(0.67-0.87)$ & 44.6 & 31.6 & 24.9 & 21.3 & 19.7 \\
\hline $\mathrm{D}$ & $271(7.3)$ & $0.25(0.21-0.32)$ & 24.7 & 18.1 & 13.3 & 11.1 & 10.1 \\
\hline
\end{tabular}


Table 3. Continued

\begin{tabular}{|c|c|c|c|c|c|c|c|}
\hline \multirow{2}{*}{ Variable } & \multirow{2}{*}{ Value } & \multirow{2}{*}{$\begin{array}{c}\text { Median OS } \\
\text { (years, } 95 \% \mathrm{Cl} \text { ) }\end{array}$} & \multicolumn{5}{|c|}{ Year } \\
\hline & & & 1 & 2 & 3 & 4 & 5 \\
\hline \multicolumn{8}{|c|}{$\begin{array}{l}\text { Initial treatment modalities ( } n=4,496 \text {, missing } \\
\text { values=76) }\end{array}$} \\
\hline Surgical resection & $888(19.8)$ & NR (NR-NR) & 93.9 & 88.2 & 82.7 & 79.5 & 77.5 \\
\hline Liver transplantation & $51(1.1)$ & NR (NR-NR) & 90.2 & 78.4 & 68.6 & 64.7 & 62.2 \\
\hline Local ablation therapy & $464(10.3)$ & NR (NR-NR) & 94.8 & 89.4 & 83.0 & 77.2 & 74.2 \\
\hline Transarterial therapy & $1,675(37.3)$ & $2.53(2.38-2.86)$ & 73.8 & 57.1 & 46.3 & 39.8 & 35.5 \\
\hline Combination therapy $^{\dagger}$ & $113(2.5)$ & $3.60(2.85-N R)$ & 69.9 & 61.9 & 57.5 & 47.8 & 45.0 \\
\hline Systemic therapy & $211(4.7)$ & $0.35(0.32-0.40)$ & 18.0 & 8.1 & 6.6 & 4.7 & 4.7 \\
\hline External beam radiation therapy & $60(1.3)$ & $0.59(0.50-0.80)$ & 26.7 & 16.7 & 10.0 & 10.0 & 10.0 \\
\hline Best supportive care & $857(19.1)$ & $0.31(0.28-0.34)$ & 28.1 & 20.4 & 16.1 & 13.2 & 12.0 \\
\hline Miscellaneous therapies ${ }^{\ddagger}$ & $177(3.9)$ & $1.38(0.98-1.86)$ & 55.4 & 40.1 & 34.5 & 31.6 & 30.4 \\
\hline
\end{tabular}

The values are expressed as the median (interquartile range) or number (\%).

OS, overall survival; $\mathrm{Cl}$, confidence interval; HBV, hepatitis B virus; HCV, hepatitis C virus; NR, not reached; UICC, Union for International Cancer Control; BCLC, Barcelona Clinic Liver Cancer.

"Patients co-infected with HBV and HCV ( $n=87)$ were also included; ${ }^{\dagger}$ Combination therapy was defined as a combined treatment with local ablation therapy and transarterial therapy; ${ }^{\ddagger}$ Miscellaneous therapies were defined as unclassifiable treatment modalities (i.e., combination therapies other than transarterial therapy and local ablation therapy).

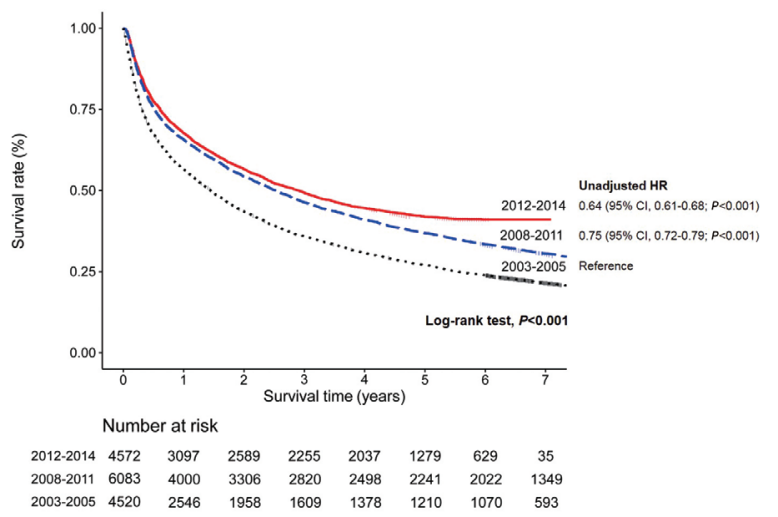

Figure 2. Overall survival curves for Korean patients with hepatocellular carcinoma in 2012-2014, 2008-2011, and 2003-2005. HR, hazard ratio; Cl, confidence interval.

\section{DISCUSSION}

We have presented representative HCC data of Koreans regarding clinical and tumor characteristics, initial treatment modalities, and OS between 2012 and 2014. As the present study was conducted using KPLCR data as part of a continuous study that serially collected Korean HCC data, comparative analyses between the data of 2012-2014 and 2008-2011 were possible. Although about $40 \%$ of the HCC patients were diagnosed at very early or early stage (BCLC stage 0 or A), another half of the HCC patients (46.7\%) were diagnosed at advanced stages (BCLC stage C or D). The proportion of patients who underwent transarterial therapy as an initial treatment reduced overtime, but it was still the most commonly performed initial treatment for HCC. Compared to the previous periods (2008-2011 cohort or 2003-2005 cohort), the OS of the 2012-2014 cohort had improved significantly, with a 5 -year OS rate of $42 \%$.

The most prominent change in HCC etiology was that the prevalence of HBV-related HCC significantly decreased compared to 2008-2011. This may have occurred in conjunction with the increase in HCC patients according to different etiologies other than viral hepatitis or alcohol. HCC originating from non-alcoholic fatty liver disease (NAFLD) has shown an increasing trend worldwide. NAFLD is a disease spectrum that starts from simple steatosis, progresses to nonalcoholic steatohepatitis, and to cirrhosis, which has a high chance of developing HCC. Moreover, a recent study has shown that dysregulation of lipid metabolism in NAFLD causing a selective loss of intrahepatic $\mathrm{CD}^{+} \mathrm{T}$ lymphocytes 

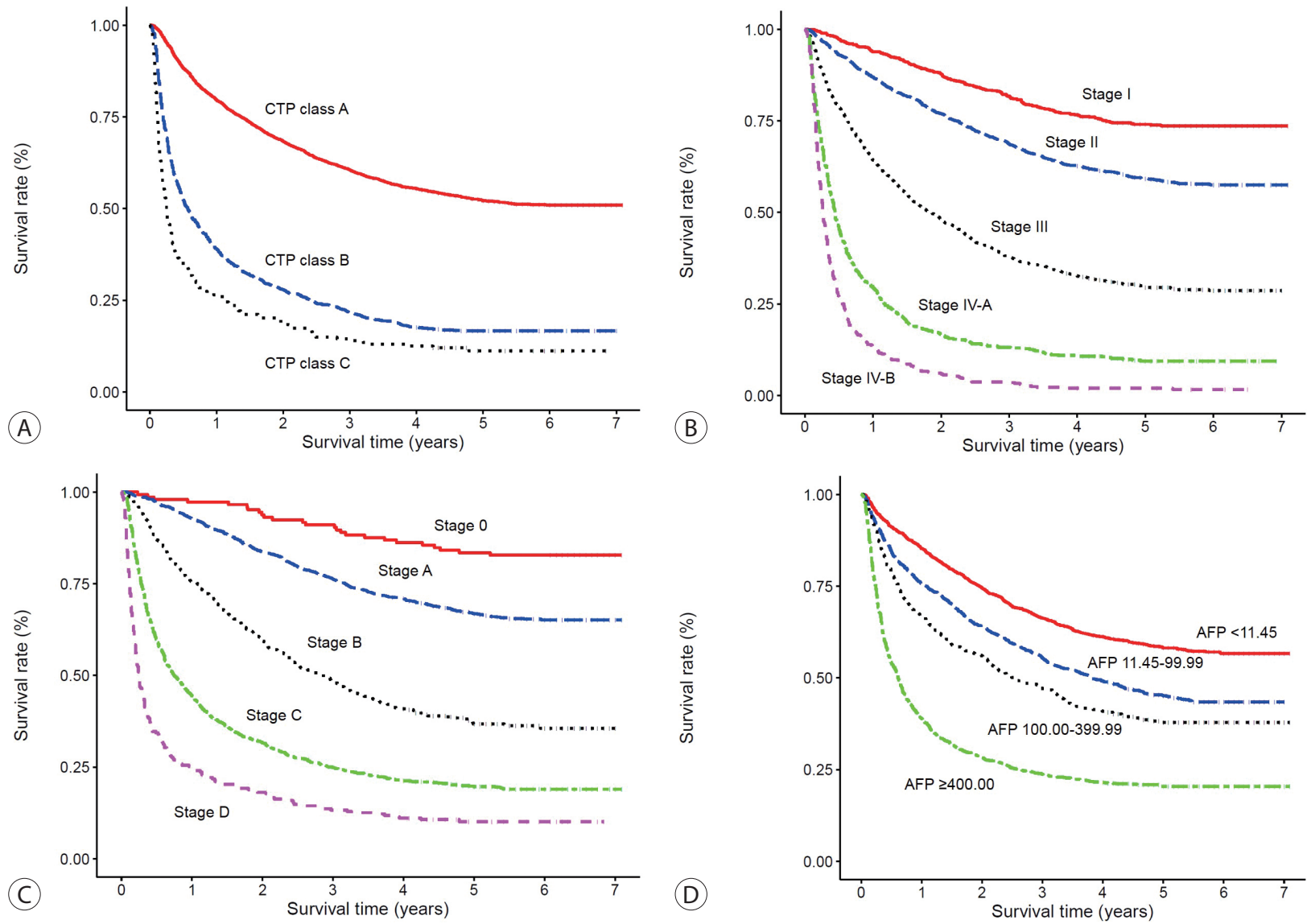

Figure 3. Overall survival curves for the subgroups divided by the Child-Turcotte-Pugh classes. (A), modified Union for International Cancer Control stages (B), Barcelona Clinic Liver Cancer stages (C), and the serum levels of alpha-fetoprotein (D). CTP, Child-Turcotte-Pugh; AFP, alpha-fetoprotein.

can directly contribute to HCC initiation and progression. ${ }^{19}$ Research on the prevalence of NAFLD in the general population in Korea is limited; however, the prevalence of NAFLD diagnosed by ultrasound from health check-ups is known to be $16-33 \%{ }^{20-22}$ NAFLD is known to accompany other metabolic diseases such as obesity and diabetes, both of which are showing steadily increasing prevalence rates (obesity, ${ }^{23} 29.7 \%$ in 2009 to $32.4 \%$ in 2015 ; diabetes, ${ }^{24} 10.1 \%$ in 2010 to $14.4 \%$ in 2016).

Compared to HCC patients in the 2008-2011 cohort, those in the 2012-2014 cohort had better liver function, with more patients having CTP class A and low MELD scores. This phenomenon may also be due to the generalization of antiviral treatment with the expanded reimbursement of drugs. By inhibiting viral replication, antiviral drugs reduce liver inflammation and fibrosis, leading to preserved liver function and decreased cirrhosis-related complications. However, the maximum tumor diameter increased significantly compared to the previous period. As previously mentioned, the proportion of patients with HCC of other etiologies (e.g., NAFLD) increased. Patients with HCC of other etiologies do not tend to undergo regular HCC surveillance and tend to be diagnosed later with large size HCC. In a study conducted by Marrero et $\mathrm{al}^{25}$, patients with cryptogenic HCC presented larger maximum tumor diameters than those with viral or alcohol-related HCC. Despite the national HCC surveillance program, the proportion of patients with advanced stage HCC (BCLC stage C or D, 46.7\%), who might not be candidates for potentially curative treatment, increased compared to the previous period (2008-2011). Therefore, further efforts should be made to improve the scale and quality of the HCC surveillance program for early detection of HCC. Serum AFP 
tests and ultrasound, which are currently being performed as part of the national cancer screening project, should be thoroughly integrated and monitored. Specifically, careful attention and optimized surveillance strategies should be implemented among patients who are prone to surveillance failure (such as those with advanced liver fibrosis, high AFP levels, or diabetes mellitus). ${ }^{7}$ Another reason for the increased proportion of patients with advanced stage HCC may be partly related to the poorer performance status accompanied by older age in the 2012-2014 cohort.

Of note, transarterial therapy was the most commonly used modality as initial treatment. In addition to patients with HCC of BCLC stage B who are indicated according to the international guidelines, transarterial therapy is the most widely used treatment modality among patients with HCC of BCLC stage A or C. In patients with BCLC stage A who are not subject to surgery or patients with BCLC stage $\mathrm{C}$ with minimal vessel invasion and relatively preserved liver function, transarterial therapy is still considered to be an effective treatment if technically accessible. However, compared to the 2008-2011 cohort, the proportion of patients receiving transarterial therapy has decreased. In contrast, the proportion of patients receiving potentially curative treatments such as surgical resection or liver transplantation has significantly increased. The proportions of patients receiving systemic therapy and radiation therapy have also increased. In short, although the overall rate of adherence to the treatment guideline is relatively low, the rates of adherence to the BCLC treatment guidelines among both patients with early and advanced stage diseases have increased (Supplementary Table 1). Considering the trend toward increasing proportion of radiation and combination therapy in this cohort, and the recent introduction of new systemic therapeutic agents (e.g., lenvatinib, nivolumab, regorafenib, and atezolizumab plus bevacizumab) in real-world practice, it is expected that HCC treatment will be more diversified and improved in the future.

It is very encouraging to witness an increasing OS of HCC patients over the study period (compared to either the 20032005 or 2008-2011 cohort). This may be partly due to the decreased rate of best supportive care and improved HCC treatment result. For instance, surgery as a curative treatment was performed increasingly even among patients with BCLC stages B and C. In a previous study, hepatic resection was well tolerated with low mortality and survival benefit, necessitating the stratification of patients and optimization of treatment among patients with HCC of BCLC stage B and C who are highly heterogenous. ${ }^{26}$ The presence and level of vascular invasion and malignant portal vein thrombosis is an important determinant for surgery. In addition to these tumor factors, preserved liver function is another important factor, and in our cohort of patients with HCC of BCLC stages B and C, more than $90 \%$ of patients undergoing surgical resection were confirmed to have CTP class A. Therefore, the increased proportion of HCC patients with preserved liver function (CTP class A) in the 2012-2014 cohort may be associated with increased survival. In the subgroup analyses, the OS of HCC patients significantly differed according to the baseline serum AFP level (Fig. 3D). In our previous study, not only the AFP level at HCC diagnosis but also a high AFP level at baseline or 6 months before diagnosis was predictive of first HCC detection at advanced stage. ${ }^{7}$ Collectively, AFP is a valuable baseline predictor of HCC, and thus, serial testing and monitoring are warranted in patients with a high risk for HCC.

The present study had several limitations. First, this cohort does not represent all Korean HCC patients because those included in this cohort are patients who received care and treatment at the hospital. However, data regarding patients who received in-hospital care are the most representative information regarding HCC treatment statuses nationwide. Second, there were significant levels of missing data of critical variables such as performance status or BCLC stage. Nevertheless, there were no significant differences in terms of demographic variables and HCC stages among those with available performance status data and those without. Lastly, important data regarding second-line treatment or antiviral treatment in patients with viral hepatitis were not collected. These factors related to the prognosis of HCC patients have been subsequently investigated in the KPLCR and, therefore, will be incorporated in future research.

In conclusion, the OS of HCC patients registered in the 
KPLCR between 2012 and 2014 significantly improved compared to the previous period. Nevertheless, as about half of the patients were still diagnosed with HCC at an advanced stage, vigorous and optimized HCC screening strategies should be implemented. Although transarterial therapy is the most widely used, various attempts at treatment diversification should be made.

\section{AUTHORSHIP STATEMENT}

1) Jeong-Hoon Lee and Young Eun Chon take responsibility for the integrity of the work as a whole, from inception to published article.

2) Specific author contributions: Conception: Young Eun Chon, Han Ah Lee, Jun Sik Yoon and Jeong-Hoon Lee; study design: Young Eun Chon, Han Ah Lee, Jun Sik Yoon and Jeong-Hoon Lee; participation in patient management and data collection: Jun Sik Yoon, Han Ah Lee, Jun Yong Park, Bo Hyun Kim, In Joon Lee, Suk Kyun Hong, Dong Hyeon Lee, Hyun-Joo Kong, Young-Joo Won, Eunyang Kim, Young Eun Chon, and Jeong-Hoon Lee; contribution to the data acquisition, responsibility for writing the paper, and statistical analysis: Young Eun Chon, Han Ah Lee, Jun Sik Yoon, and Jeong-Hoon Lee.

3) All authors have reviewed the paper and approved the final version.

\section{ACKNOWLEDGMENTS}

This study was supported by Korean Liver Cancer Association Research Award (recipient: J. H. L.). The database used in this study was provided by the Korean Central Cancer Registry, Ministry of Health and Welfare, South Korea, and the Korean Liver Cancer Association.

\section{SUPPLEMENTARY MATERIAL}

Supplementary data can be found with this article online http://www.e-jlc.org/html/https://doi.org/10.17998/ jlc.20.2.135.

\section{Conflicts of Interest}

The authors have no conflicts to disclose.

\section{REFERENCES}

1. Sayiner M, Golabi P, Younossi ZM. Disease burden of hepatocellular carcinoma: a global perspective. Dig Dis Sci 2019;64:910-917.

2. El-Serag HB, Rudolph KL. Hepatocellular carcinoma: epidemiology and molecular carcinogenesis. Gastroenterology 2007;132:25572576.

3. National Cancer Center. Annual report of cancer statistics in Korea in 2016. Goyang: National Cancer Center; 2018. 1-221.

4. Kim BH, Park JW. Epidemiology of liver cancer in South Korea. Clin Mol Hepatol 2018;24:1-9.

5. Cho EJ, Kim SE, Suk KT, An J, Jeong SW, Chung WJ, et al. Current status and strategies for hepatitis B control in Korea. Clin Mol Hepatol 2017;23:205-211.

6. Choi J, Han S, Kim N, Lim YS. Increasing burden of liver cancer despite extensive use of antiviral agents in a hepatitis B virus-endemic population. Hepatology 2017;66:1454-1463.

7. Chon YE, Jung KS, Kim MJ, Choi JY, An C, Park JY, et al. Predictors of failure to detect early hepatocellular carcinoma in patients with chronic hepatitis B who received regular surveillance. Aliment Pharmacol Ther 2018;47:1201-1212.

8. Kwon JW, Tchoe HJ, Lee J, Suh JK, Lee JH, Shin S. The impact of national surveillance for liver cancer: results from real-world setting in Korea. Gut Liver 2020;14:108-116.

9. Suh M, Song S, Cho HN, Park B, Jun JK, Choi E, et al. Trends in Participation Rates for the National Cancer Screening Program in Korea, 2002-2012. Cancer Res Treat 2017;49:798-806.

10. Marrero JA, Kulik LM, Sirlin CB, Zhu AX, Finn RS, Abecassis MM, et al. Diagnosis, staging, and management of hepatocellular carcinoma: 2018 practice guidance by the American Association for the Study of Liver Diseases. Hepatology 2018;68:723-750.

11. European Association For The Study Of The Liver; European Organisation For Research And Treatment Of Cancer. EASL-EORTC clinical practice guidelines: management of hepatocellular carcinoma. J Hepatol 2012;56:908-943.

12. Kim DY, Kim HJ, Han KH, Han SY, Heo J, Woo HY, et al. Real-life experience of sorafenib treatment for hepatocellular carcinoma in Korea: from GIDEON data. Cancer Res Treat 2016;48:1243-1252.

13. Choi SH, Seong J. Strategic application of radiotherapy for hepatocellular carcinoma. Clin Mol Hepatol 2018;24:114-134.

14. Yoon JS, Lee HA, Park JY, Kim BH, Lee IJ, Chon YE, et al. Hepatocellular carcinoma in Korea between 2008 and 2011: an analysis of Korean Nationwide Cancer Registry. J Liver Cancer 2020;20:41-52.

15. Sheu JC, Sung JL, Chen DS, Yang PM, Lai MY, Lee CS, et al. Growth rate of asymptomatic hepatocellular carcinoma and its clinical implications. Gastroenterology 1985;89:259-266. 
16. Kudo M, Kitano M, Sakurai T, Nishida N. General rules for the clinical and pathological study of primary liver cancer, nationwide follow-up survey and clinical practice guidelines: the outstanding achievements of the Liver Cancer Study Group of Japan. Dig Dis 2015:33:765-770.

17. Llovet JM, Brú C, Bruix J. Prognosis of hepatocellular carcinoma: the BCLC staging classification. Semin Liver Dis 1999;19:329-338.

18. Lim YS, Sinn DH, Kim SH, Korea Central Cancer Registry, Hwang JS, Han KH. Characteristics and survival of Korean patients with hepatocellular carcinoma: a nationwide random sample study. J Liver Cancer 2014;14:97-107.

19. $M a ~ C$, Kesarwala AH, Eggert $T$, Medina-Echeverz J, Kleiner $D E$, Jin $P$, et al. NAFLD causes selective CD4(+) T lymphocyte loss and promotes hepatocarcinogenesis. Nature 2016;531:253-257.

20. Park SH, Jeon WK, Kim SH, Kim HJ, Park DI, Cho YK, et al. Prevalence and risk factors of non-alcoholic fatty liver disease among Korean adults. J Gastroenterol Hepatol 2006;21(1 Pt 1):138-143.

21. Choi SY, Kim D, Kim HJ, Kang JH, Chung SJ, Park MJ, et al. The relation between non-alcoholic fatty liver disease and the risk of coronary heart disease in Koreans. Am J Gastroenterol 2009;104:1953-
1960.

22. Lee K, Sung JA, Kim JS, Park TJ. The roles of obesity and gender on the relationship between metabolic risk factors and non-alcoholic fatty liver disease in Koreans. Diabetes Metab Res Rev 2009;25:150155.

23. Seo MH, Lee WY, Kim SS, Kang JH, Kang JH, Kim KK, et al. 2018 Korean Society for the study of obesity guideline for the management of obesity in Korea. J Obes Metab Syndr 2019;28:40-45.

24. Kim BY, Won JC, Lee JH, Kim HS, Park JH, Ha KH, et al. Diabetes fact sheets in Korea, 2018: an appraisal of current status. Diabetes Metab J 2019;43:487-494.

25. Marrero JA, Fontana RJ, Su GL, Conjeevaram HS, Emick DM, Lok AS. NAFLD may be a common underlying liver disease in patients with hepatocellular carcinoma in the United States. Hepatology 2002;36:1349-1354.

26. Torzilli G, Donadon M, Marconi M, Palmisano A, Del Fabbro D, Spinelli $A$, et al. Hepatectomy for stage $B$ and stage $C$ hepatocellular carcinoma in the Barcelona Clinic Liver Cancer classification: results of a prospective analysis. Arch Surg 2008;143:1082-1090. 
Journal of Liver Cancer

Volume 20 Number 2, September 2020

Supplementary Table 1. Rates of adherence to the BCLC treatment guideline in each BCLC stage and the total study population

\begin{tabular}{lc}
\hline BCLC stage & Value (2012-2014) \\
\hline O or A & $817 / 1,491(54.8)$ \\
B & $253 / 459(55.1)$ \\
C & $170 / 1,451(11.7)$ \\
D & $161 / 264(61.0)$ \\
Total & $1,401 / 3,665(38.2)$ \\
\hline BCLC stage & Value (2008-2011) \\
\hline O or A & $1,277 / 2,695(47.4)$ \\
B & $407 / 648(62.8)$ \\
C & $180 / 1,899(9.5)$ \\
D & $258 / 381(67.7)$ \\
Total & $2,122 / 5,623(37.7)$ \\
\hline The data are presented as number (\%). \\
BCLC, Barcelona Clinic Liver Cancer.
\end{tabular}




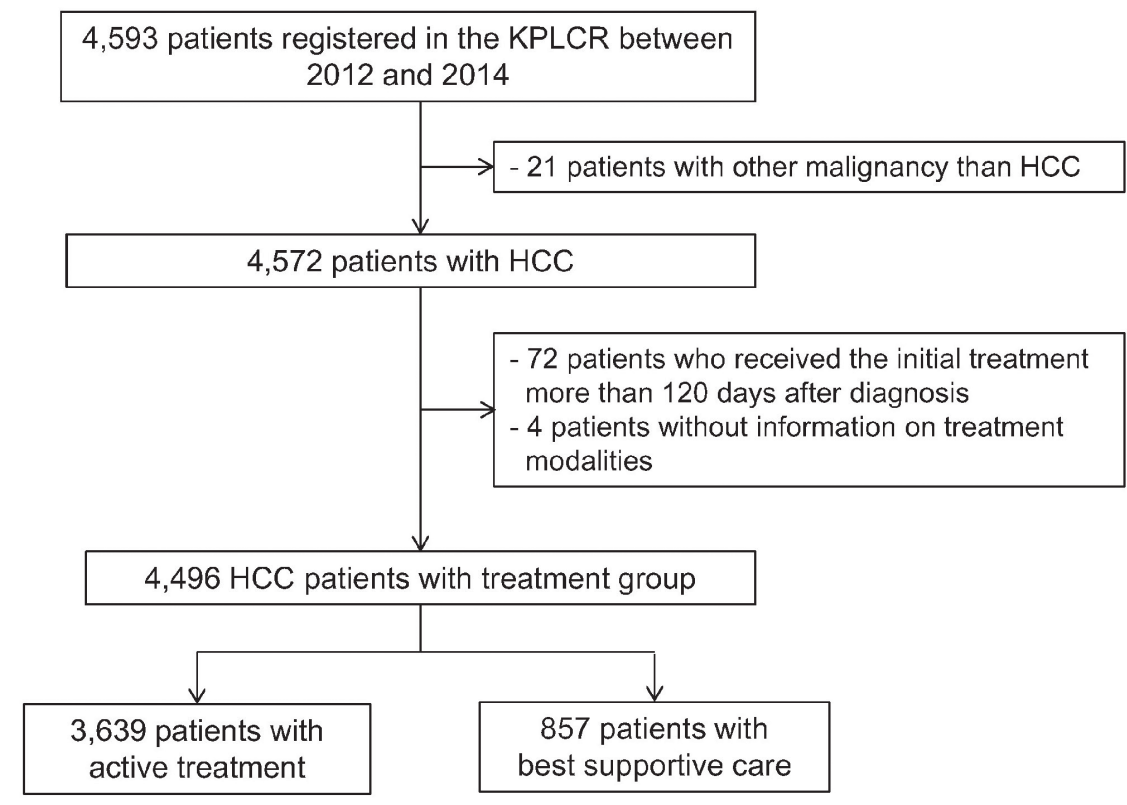

Supplementary Figure 1. Flow chart of patient selection in the present study. KPLCR, Korean Primary Liver Cancer Registry; HCC, hepatocellular carcinoma. 